\title{
Comparison of the Apnea-Hypopnea Index Determined by a Peripheral Arterial Tonometry-Based Device With That Determined by Polysomnography \\ - Results From a Multicenter Study -
}

\author{
Takatoshi Kasai, MD, PhD; Yoshifumi Takata, MD; Akiomi Yoshihisa, MD, PhD; \\ Yasuchika Takeishi, MD, PhD; Kazuo Chin, MD, PhD; Shin-ichi Ando, MD, PhD; \\ Fusae Kawana,BSc; Shin-ichi Momomura, MD
}

\begin{abstract}
Background: Sleep disordered breathing (SDB) is prevalent and associated with increased mortality in patients both with and without cardiovascular disease (CVD). Many portable monitoring devices, including peripheral arterial tonometry (PAT)-based devices, have been developed. Although previous studies have demonstrated that the apnea-hypopnea index (AHI) determined by PAT devices (pAHI) is strongly correlated with AHI determined by polysomnography (AHI-PSG), no data have been reported from a Japanese patient population or patients with CVD. In this study we compared the parameters determined by PAT-based devices with those determined by polysomnography in Japanese patients with CVD.
\end{abstract}

Methods and Results: We enrolled 120 patients undergoing overnight polysomnography at 6 Japanese centers. A PAT-based device was used simultaneously with polysomnography. Polysomnography recordings were scored centrally by a technician in a blinded manner. PAT-based device recordings were scored using an automatic algorithm. There was a strong correlation between pAHI and AHI-PSG $(r=0.896 ; P<0.001)$ with acceptable agreement. The strong correlation between $p A H I$ and AHI-PSG was observed in patients with CVD $(n=55 ; P=0.849 ; P<0.001)$ and without CVD $(n=65 ; r=0.927 ; P<0.001)$. The presence or absence of CVD did not affect the relationship between $\mathrm{PAHI}$ and AHI-PSG $(\mathrm{P}=0.225)$.

Conclusions: A PAT-based device provides a reliable AHI in a Japanese patient population, even in patients with CVD. These findings may help reduce the number of patients with undiagnosed SDB and CVD.

Key Words: Cardiovascular disease; Polygraphy; Sleep disordered breathing; Sympathetic nervous activity

$\mathbf{S}$ leep disordered breathing (SDB) is a common disorder and has a high risk of cardiovascular morbidity and mortality. ${ }^{1}$ In addition, SDB is more prevalent in patients with than without cardiovascular diseases (CVDs), and it is associated with poor clinical outcomes. ${ }^{1}$ However, it has been estimated that at least $80 \%$ of moderate-tosevere SDB remains undiagnosed in middle-aged adults in the US, 2,3 particularly in patients with CVD because of the limited awareness of the importance of SDB in CVD among physicians and patients. Although in-laboratory overnight polysomnography is the gold standard examina- tion for $\mathrm{SDB},{ }^{4}$ it is costly, labor intensive, has limited accessibility, and often has long waiting times. ${ }^{5}$ Alternative methods that may facilitate the diagnosis of SDB are needed to resolve these issues. Thus, portable monitoring devices (i.e., polygraphy) have been developed in order to increase accessibility and reduce the costs of diagnosing SDB. ${ }^{6}$

One polygraphy device is the peripheral arterial tonometry (PAT)-based device. Many studies have demonstrated that the respiratory parameters determined by a PAT-based device are well correlated with those determined by formal

Received August 25, 2020; accepted August 25, 2020; J-STAGE Advance Publication released online October 16, 2020 Time for primary review: 1 day

Cardiovascular Respiratory Sleep Medicine (T.K., F.K.), Department of Cardiovascular Medicine (T.K.), Juntendo University Graduate School of Medicine, Tokyo; Department of Cardiology, Tokyo Medical University, Tokyo (Y. Takata); Department of Cardiovascular Medicine, Fukushima Medical University, Fukushima (A.Y., Y. Takeishi); Department of Respiratory Care and Sleep Control Medicine, Graduate School of Medicine, Kyoto University, Kyoto (K.C.); Sleep Apnea Center, Kyushu University Hospital, Fukuoka (S.A.); and Department of Cardiovascular Medicine, Saitama Medical Center, Jichi Medical University, Saitama (S.M.), Japan

Mailing address: Takatoshi Kasai, MD, PhD, Department of Cardiovascular Medicine, Juntendo University Graduate School of Medicine, 2-1-1 Hongo, Bunkyo-ku, Tokyo 113-8421, Japan. E-mail: kasai-t@mx6.nisiq.net

All rights are reserved to the Japanese Circulation Society. For permissions, please e-mail: cr@j-circ.or.jp

ISSN-2434-0790 
polysomnography. ${ }^{7-9}$ Although a meta-analysis indicated that there was a very good correlation between the severity of SDB determined by PAT-based devices and that determined by polysomnography in various populations, ${ }^{10}$ there are no specific data comparing the parameters determined by PAT-based devices with those simultaneously determined by polysomnography in a Japanese patient population. Furthermore, the PAT-based device uses the PAT signal, pulse rate, oxyhemoglobin saturation $\left(\mathrm{SO}_{2}\right)$, and actigraphy ${ }^{\mathbf{1 1}}$ to measure SDB, and these parameters may be affected by altered vascular properties ${ }^{12}$ or cardiac rhythms or the presence of underlying CVD and the use of related medications. However, there are limited data comparing the parameters determined by PAT-based devices with those determined by polysomnography in patients with CVD. The specific hypothesis we tested in the present study is that the severity of SDB determined by PAT-based device is well correlated with that determined simultaneously by polysomnography in Japanese individuals with and without CVD.

Therefore, the aim of the study was to assess the correlations and agreements of parameters determined by PATbased devices with those determined simultaneously by polysomnography in a Japanese patient population, including patients with CVD. In addition, we assessed the correlations and agreements of the parameters determined by PAT-based devices with those determined simultaneously by polysomnography separately in patients with and without CVD to investigate whether the correlations of the parameters in patients with CVD are comparable to those in patients without CVD.

\section{Methods}

\section{Subjects}

We prospectively enrolled adult patients (age $\geq 20$ years) who were hospitalized for overnight polysomnography with a suspicion of SDB between September 2014 and May 2015 at 6 Japanese centers: Juntendo University Hospital (Tokyo, Japan), Tokyo Medical University Hospital (Tokyo, Japan), Fukushima Medical University Hospital (Fukushima, Japan), Kyoto University Hospital (Kyoto, Japan), Kyushu University Hospital (Fukuoka, Japan), and Saitama Medical Center of the Jichi Medical University (Saitama, Japan). The exclusion criteria were as follows: (1) patients with a history of permanent pacemaker, implantable cardioverter defibrillator, or cardiac resynchronization therapy; (2) patients using $\alpha$-blockers within $24 \mathrm{~h}$ prior to polysomnography (patients using carvedilol, an $\alpha / \beta$-blocker, were not excluded); (3) patients using shortacting nitrates within $8 \mathrm{~h}$ prior to polysomnography; (4) patients with advanced chronic obstructive pulmonary disease, cor pulmonale, end-stage renal disease, hepatic failure, neuromuscular diseases, post-stroke with neurological deficit, ventilation for hypercapnia, or supplemental oxygen; and (5) patients who had difficulty attaching the PAT sensor to their fingers.

The protocol of this multicenter study was approved by each institution's research ethics board, and the study complied with the ethical principles of the Declaration of Helsinki. All patients provided written informed consent for the use of their data, with all identifying information removed.

\section{Polysomnography}

All patients underwent overnight polysomnography using a digital polygraph system (Alice; Philips Respironics, Murrysville, PA, USA) following the standard procedure recommended by the American Academy of Sleep Medicine (AASM). ${ }^{13}$ All digital files for overnight polysomnography were sent to the core laboratory in the Juntendo University and scored centrally by an experienced registered sleep technician who was blinded to the patients' characteristics and data from the PAT-based device. Definitions and scoring methods were based on the AASM Manual version 2.2. ${ }^{13}$ Apneas were classified as obstructive or central according to the presence or absence of thoracoabdominal motion, respectively. Hypopneas were classified as central if none of the following criteria were met: snoring during the event, increased inspiratory flattening of the nasal pressure compared with baseline breathing, and associated thoracoabdominal paradox during but not before the event. Apneas and hypopneas were quantified, and SDB severity was assessed using the frequency of apneas and hypopneas per hour of sleep (i.e., apnea-hypopnea index [AHI]). Patients were divided into categories according to AHI as follows: no $\mathrm{SDB}, \mathrm{AHI}<5$; mild $\mathrm{SDB}, 5 \leq \mathrm{AHI}<15$; moderate $\mathrm{SDB}, 15 \leq \mathrm{AHI}<30$; severe $\mathrm{SDB}, \mathrm{AHI} \geq 30{ }^{14}$

\section{PAT-Based Device}

The same type and version of PAT-based device (WatchPAT 200; Itamar Medical, Caesarea, Israel) was used for each patient. The device is worn around the wrist and has 2 finger probes, one for a PAT sensor and the other for an oximetry sensor. The wrist unit contains the sensor for measuring wrist movements through an accelerometer. Thus, information regarding changes in PAT, pulse oximetry, pulse rate, and actigraphy can be recorded. The PAT signal is measured with a plethysmographic-based finger-mounted probe and represents arterial pulsatile volume changes of the finger that are regulated by $\alpha$-adrenergic innervation of the smooth muscles of the vasculature of the finger, ${ }^{15}$ and thus reflect sympathetic nervous system activity. This augmentation in sympathetic nervous activity is seen as a reduction in PAT amplitude accompanied by an increase in pulse rate and desaturation at the termination of respiratory events. The detailed algorithms of sleep/wake detection using the accelerometer and sleep staging using the PAT signal have been described previously. ${ }^{16-18}$ In this study, all PAT-based device recordings were downloaded and sent to the core laboratory, where all data were analyzed automatically in an offline application (Zzz PAT version 4.3.62; Itamar Medical). PAT-based device determinations of AHI (pAHI), mean and minimum $\mathrm{SO}_{2}$, total sleep time (TST), percentage of rapid eye movement (REM) sleep, and light (counterpart of sleep stage N1+2) and deep (counterpart of sleep stage N3) non-REM sleep were computed automatically.

\section{Study Protocol and Collection of Other Data}

The study was a multicenter prospective study comparing the automatically scored pAHI with manually scored polysomnography-determined AHI (AHI-PSG) by simultaneously using a PAT device and polysomnography. In addition to the regular polysomnography setup and protocol, PAT-based devices were placed on patients' hands, and recordings from both monitors started simultaneously. Patients with a TST on polysomnography of $<180 \mathrm{~min}$ or whose polysomnographic recording and/or PAT-based 
device recordings had an issue or poor quality were excluded from analyses.

Demographic and medical information, including medications, was collected for all patients from clinical charts and/or by interview at the time of polysomnography. In particular, a history of CVD was confirmed by the attending cardiologists.

\section{Statistical Analysis}

Data are presented as the mean $\pm \mathrm{SD}$ or median with interquartile range (IQR) for continuous variables and as ratios $(\%)$ for categorical variables. Polysomnography data and PAT-based device data were compared using paired t-tests and the Wilcoxon signed-rank test for continuous variables. Correlations between the polysomnography and PAT-based device data were assessed based on Pearson or Spearman correlation coefficients, as appropriate. BlandAltman plots were used to assess mean differences and

\begin{tabular}{|lc|}
\hline Table 1. Patient Characteristics $(\mathbf{n}=\mathbf{1 2 0})$ & \\
Age (years) & $58.0 \pm 11.9$ \\
Male sex & $102(85.0)$ \\
$\mathrm{BMI}\left(\mathrm{kg} / \mathrm{m}^{2}\right)$ & $26.4 \pm 5.4$ \\
$\mathrm{AHI}(/ \mathrm{h})$ & $31.7 \pm 20.6$ \\
Obstructive AHI $/ \mathrm{h})$ & $22.4[24.4]$ \\
Central AHI $(/ \mathrm{h})$ & $1.6[5.0]$ \\
SDB severity & \\
None & $6(5.0)$ \\
Mild & $21(17.5)$ \\
Moderate & $32(26.7)$ \\
Severe & $61(50.8)$ \\
Diabetes & $25(20.8)$ \\
Hypertension & $72(60.0)$ \\
CVD & $55(45.8)$ \\
HF & $29(24.2)$ \\
Coronary artery disease & $24(20.0)$ \\
Atrial fibrillation & $21(17.5)$ \\
ACEls/ARBs & $55(45.8)$ \\
$\beta$-blockers & $41(34.2)$ \\
Carvedilol & $18(15.0)$ \\
Calcium channel blockers & $39(32.5)$ \\
\hline
\end{tabular}

Data are presented as the mean $\pm \mathrm{SD}$, median [interquartile range], or as $n(\%)$. ACEls, angiotensin-converting enzyme inhibitors; $\mathrm{AHI}$, apnea-hypopnea index; ARBs, angiotensin II receptor blockers; BMI, body mass index; CVD, cardiovascular disease; SDB, sleep disordered breathing. limits of agreement. To assess the discriminatory power of the pAHI to predict severe SDB (i.e., AHI-PSG $\geq 30 / h$ ), we computed the area under the curve (AUC) using receiver operating characteristic (ROC) analyses. The best cut-off value of $\mathrm{pAHI}$ for predicting AHI-PSG $\geq 30 / \mathrm{h}$ was identified as the value that minimized the expression $\left[\left(1-{\text { sensitivity })^{2}}^{2}\right.\right.$ $\left.+(1-\text { specificity })^{2}\right] .{ }^{19}$ To compare the correlation slopes between patients with and without CVD, the relationship between pAHI and AHI-PSG was analyzed with the interaction between the subgroups. Two-tailed $\mathrm{P}<0.05$ was considered significant. All analyses were performed using SPSS v.23.0 (IBM Corp., Armonk, NY, USA).

\section{Results}

Overall, 128 Japanese patients were enrolled from 6 centers. We excluded 2 patients with a TST on polysomnography $<180 \mathrm{~min}, 2$ patients due to errors in data transfer/conversion, 2 patients due to poor polysomnography signals, and 2 patients due to poor PAT-based device signals. Thus, data from 120 patients were included in the final analysis. The characteristics of these 120 patients are given in Table 1. Most participants had moderate to severe SDB. Approximately half the patients had CVD, including heart failure, coronary artery disease, and atrial fibrillation (10 persistent/permanent, 11 paroxysmal). Data from polysomnography and the PAT-based devices are given in Table 2. Although pAHI and AHI-PSG were identical, the sleep duration, sleep stage, and oxygenation parameters differed significantly between the 2 methods.

A significant and strong correlation was found between pAHI and AHI-PSG (Figure 1A), and an acceptable agreement was observed (Figure 1B). The AUC was 0.890 (standard error, $0.030 ; \mathrm{P}<0.001$ ). The best cut-off of $\mathrm{pAHI}$ predicting AHI-PSG $\geq 30 / \mathrm{h}$ was $27.5 / \mathrm{h}$, with a sensitivity and specificity of $88.5 \%$ and $74.6 \%$, respectively. A moderate correlation was found between TST measured using a PAT-based device and TST measured using polysomnography $(\mathrm{r}=0.548, \mathrm{P}<0.001)$, with a mean difference of $-70.4 \mathrm{~min}$ (limits of agreement, -209.2 to $68.4 \mathrm{~min}$ ). A weak correlation was found between deep sleep measured using a PAT-based device and N3 sleep measured using polysomnography $(\mathrm{r}=0.372, \mathrm{P}<0.001)$, with a mean difference of $-6.7 \%$ (limits of agreement, $-20.7 \%$ to $7.3 \%$ ). A weak correlation was found between REM sleep measured using a PAT-based device and R sleep measured using polysomnography $(\mathrm{r}=0.280, \mathrm{P}=0.002)$, with a mean difference of $-7.5 \%$ (limits of agreement, $-25.5 \%$ to $10.5 \%$ ). The mean $\mathrm{SO}_{2}$ measured using a PAT-based device was significantly correlated with the mean $\mathrm{SO}_{2}$ measured using polysom-

\begin{tabular}{|lccc|}
\hline \multicolumn{2}{|l|}{ Table 2. Data From PAT-Based Devices and Polysomnography in All Patients $(\mathbf{n}=\mathbf{1 2 0})$} & \\
& PAT-based device & Polysomnography & P value \\
$\mathrm{AHI}(/ \mathrm{h})$ & $32.1 \pm 18.2$ & $31.7 \pm 20.6$ & 0.575 \\
Mean $\mathrm{SO}_{2}(\%)$ & $93.9 \pm 1.9$ & $95.9 \pm 1.7$ & $<0.001$ \\
Minimum $\mathrm{SO}_{2}(\%)$ & $79.7 \pm 7.4$ & $78.5 \pm 10.2$ & 0.008 \\
Total sleep time (min) & $459.7 \pm 54.6$ & $389.8 \pm 76.9$ & $<0.001$ \\
Light sleep/Stage N1+N2 (\%) & $64.7 \pm 12.9$ & $78.9 \pm 9.7$ & $<0.001$ \\
Deep sleep/Stage N3 (\%) & $14.4[8.9]$ & $5.6[10.4]$ & $<0.001$ \\
REM/Stage R (\%) & $22.0 \pm 8.5$ & $14.5 \pm 6.3$ & $<0.001$ \\
\hline
\end{tabular}

Unless indicated otherwise, data are presented as the mean \pm SD or median [interquartile range]. AHI, apnea-hypopnea index; PAT, peripheral arterial tonometry; REM, rapid eye movement; $\mathrm{SO}_{2}$, oxyhemoglobin saturation. 
A

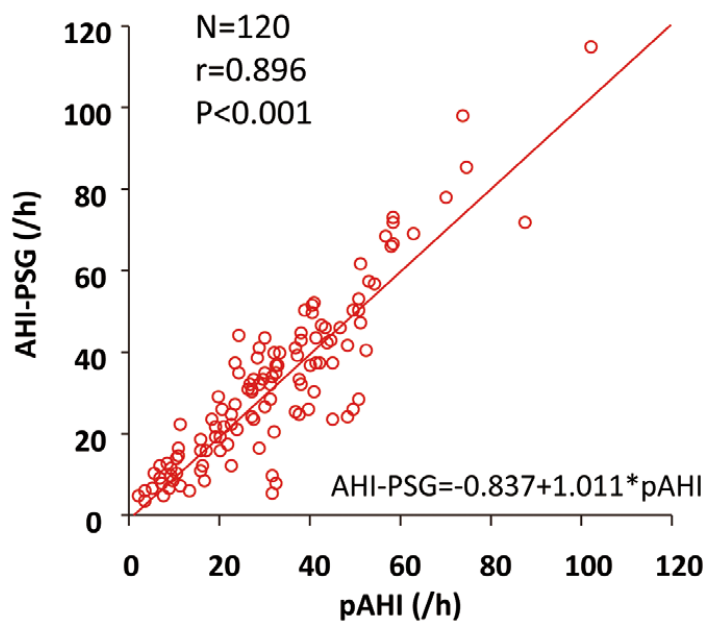

B

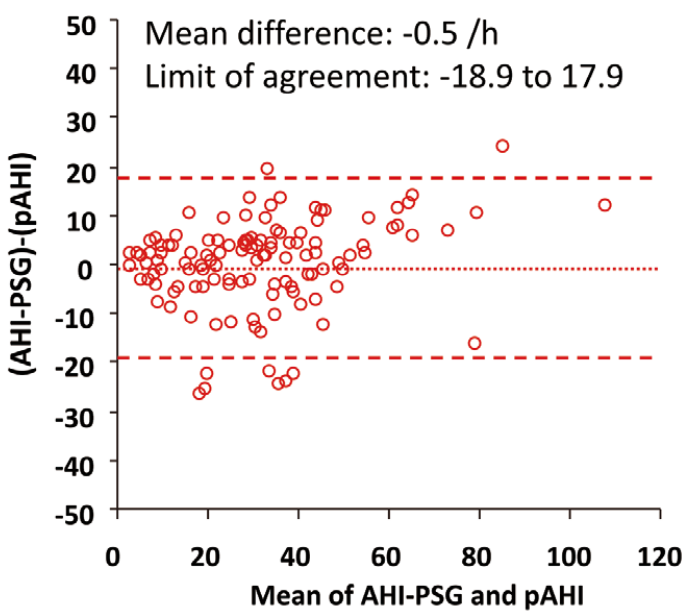

Figure 1. (A) Correlation and (B) agreement between the apnea-hypopnea index determined using a peripheral arterial tonometry-based device (pAHI) and the apnea-hypopnea index determined by polysomnography (AHI-PSG) in all patients. There was a strong correlation and between AHI-PSG and pAHI (A). There was also an agreement between AHI-PSG and pAHI (B). The dotted line represents the mean difference and the dashed lines represent the limits of agreement.

\begin{tabular}{|lccc|}
\hline \multicolumn{1}{|c|}{ Table 3. Characteristics of Patients With and Without CVD } & $\begin{array}{c}\text { Without CVD } \\
(\mathbf{n = 6 5 )}\end{array}$ & $\begin{array}{c}\text { With CVD } \\
(\mathbf{n}=55)\end{array}$ & P value \\
Age (years) & $55.2 \pm 10.3$ & $61.2 \pm 12.8$ & 0.006 \\
Male sex & $55(84.6)$ & $47(85.5)$ & 0.999 \\
BMI (kg/m $\left.{ }^{2}\right)$ & $26.4 \pm 4.9$ & $26.5 \pm 6.1$ & 0.892 \\
AHI $/ / h)$ & $29.4 \pm 21.4$ & $34.3 \pm 19.4$ & 0.111 \\
Obstructive AHI (/h) & $26.4[24.7]$ & $17.7[22.4]$ & 0.232 \\
Central AHI /h) & $1.1[2.9]$ & $3.5[16.2]$ & $<0.001$ \\
SDB severity & & & 0.100 \\
None & $5(7.7)$ & $1(1.8)$ & \\
Mild & $15(23.1)$ & $6(10.9)$ & \\
Moderate & $14(21.5)$ & $18(32.7)$ & \\
Severe & $31(47.7)$ & $30(54.5)$ & 0.006 \\
Diabetes & $7(10.8)$ & $18(32.7)$ & 0.135 \\
Hypertension & $35(53.8)$ & $37(67.3)$ & $<0.001$ \\
ACEls/ARBs & $17(27.0)$ & $38(66.7)$ & $<0.001$ \\
$\beta$-blockers & $4(6.2)$ & $37(67.3)$ & $<0.001$ \\
Carvedilol & $1(1.5)$ & $17(30.9)$ & 0.732 \\
Calcium channel blockers & $22(33.8)$ & $17(30.9)$ & \\
\hline
\end{tabular}

Unless indicated otherwise, data are presented as the mean \pm SD, median [interquartile range], or as $\mathrm{n}(\%)$. Abbreviations as in Table 1.

nography $(\mathrm{r}=0.871, \mathrm{P}<0.001)$, with a mean difference of $2.0 \%$ (limits of agreement, $-0.2 \%$ to $3.8 \%$ ). The minimum $\mathrm{SO}_{2}$ measured using a PAT-based device was significantly correlated with the minimum $\mathrm{SO}_{2}$ measured using polysomnography $(\mathrm{r}=0.894, \mathrm{P}<0.001)$, with a mean difference of $-1.2 \%$ (limits of agreement, $-11.0 \%$ to $8.6 \%$ ).

The characteristics of patients with and without CVD are given in Table 3. Patients with CVD were older, had greater central AHI, and were more likely to have diabetes and use angiotensin-converting enzyme inhibitors (ACEIs)/ angiotensin II receptor blockers (ARBs) and $\beta$-blockers, including carvedilol. The polysomnography and PAT-based device data of patients without CVD are given in Table 4. There was no significant difference between pAHI and AHI-PSG in patients without CVD. The sleep and oxygenation parameters measured in patients without CVD differed significantly between the PAT-based device and polysomnography ( $\mathrm{P}<0.001$ for all). The polysomnography and PAT-based device data of patients with CVD are given in Table 5. There was no significant difference between 


\begin{tabular}{|c|c|c|c|}
\hline & PAT-based device & Polysomnography & $P$ value \\
\hline $\mathrm{AHI}(/ \mathrm{h})$ & $29.5 \pm 18.6$ & $29.4 \pm 21.4$ & 0.951 \\
\hline Mean $\mathrm{SO}_{2}(\%)$ & $94.0 \pm 1.4$ & $96.2 \pm 1.3$ & $<0.001$ \\
\hline Minimum $\mathrm{SO}_{2}(\%)$ & $79.4 \pm 6.7$ & $78.2 \pm 11.0$ & 0.239 \\
\hline Total sleep time (min) & $459.2 \pm 48.2$ & $396.6 \pm 76.1$ & $<0.001$ \\
\hline Light sleep/Stage N1+N2 (\%) & $61.9 \pm 12.7$ & $79.1 \pm 9.7$ & $<0.001$ \\
\hline Deep sleep/Stage N3 (\%) & $15.5[6.4]$ & $5.7[11.1]$ & $<0.001$ \\
\hline REM/Stage R (\%) & $23.3 \pm 8.9$ & $14.1 \pm 6.2$ & $<0.001$ \\
\hline
\end{tabular}

Unless indicated otherwise, data are presented as the mean \pm SD or median [interquartile range]. Abbreviations as in Tables 1,2.

\begin{tabular}{|lccc|}
\hline \multicolumn{2}{|l|}{ Table 5. Data From PAT-Based Devices and Polysomnography in Patients With CVD ( $\mathbf{n}=55)$} & \\
& PAT-based device & Polysomnography & P value \\
$\mathrm{AHI}(/ \mathrm{h})$ & $35.3 \pm 17.4$ & $34.3 \pm 19.4$ & 0.497 \\
Mean $\mathrm{SO}_{2}(\%)$ & $93.8 \pm 2.4$ & $95.5 \pm 2.1$ & $<0.001$ \\
Minimum $\mathrm{SO}_{2}(\%)$ & $80.1 \pm 8.2$ & $78.5 \pm 10.4$ & 0.010 \\
Total sleep time (min) & $460.2 \pm 61.8$ & $381.8 \pm 71.1$ & $<0.001$ \\
Light sleep/Stage N1+N2 (\%) & $68.2 \pm 12.4$ & $78.9 \pm 9.7$ & $<0.001$ \\
Deep sleep/Stage N3 (\%) & $11.3[9.2]$ & $4.5[10.0]$ & $<0.001$ \\
REM/Stage R (\%) & $20.4 \pm 7.8$ & $14.9 \pm 6.5$ & $<0.001$ \\
\hline
\end{tabular}

Unless indicated otherwise, data are presented as the mean \pm SD or median [interquartile range]. Abbreviations as in Tables 1,2.

A

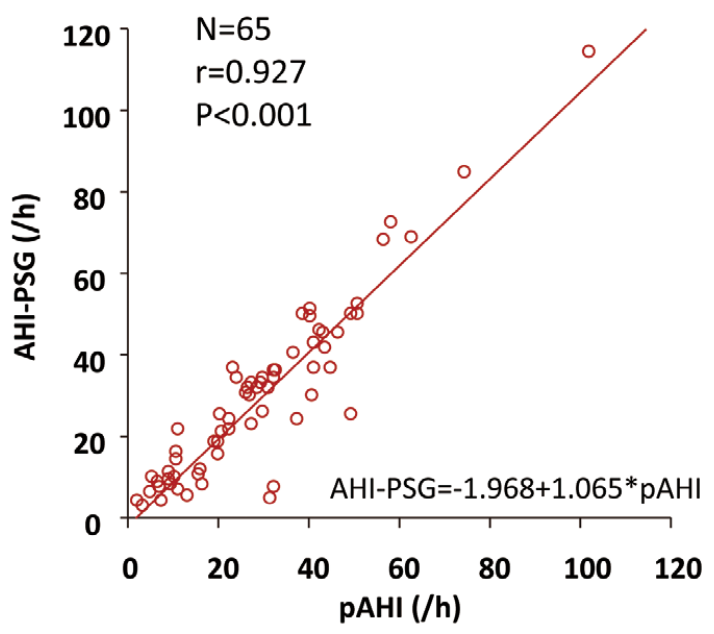

B

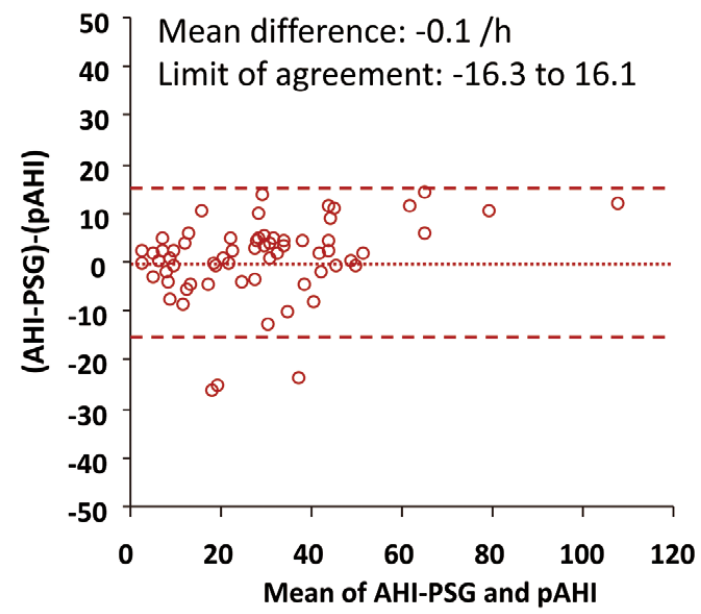

Figure 2. (A) Correlation and (B) agreement between the apnea-hypopnea index determined using a peripheral arterial tonometry-based device (pAHI) and the apnea-hypopnea index determined by polysomnography (AHI-PSG) in patients without cardiovascular disease (CVD). There was a strong correlation $(\mathbf{A})$ and agreement $(\mathbf{B})$ between AHI-PSG and pAHI in patients without CVD. The dotted line represents the mean difference and dashed lines represent the limits of agreement.

pAHI and AHI-PSG in patients with CVD. However, there were significant differences in sleep and oxygenation parameters measured in patients with CVD between a PAT-based device and polysomnography $(\mathrm{P}<0.010$ for minimum $\mathrm{SO}_{2} ; \mathrm{P}<0.001$ for remaining parameters).

There was no significant interaction between CVD and

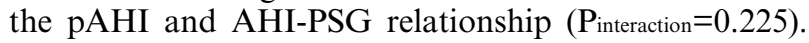

No significant interactions between CVD and TST, deep sleep/N3 sleep, or REM sleep/R sleep were observed (Pinteraction $=0.311,0.397$, and 0.678, respectively). No significant interactions between $\mathrm{CVD}$ and mean $\mathrm{SO}_{2}$ $($ Pinteraction $=0.173)$ or minimum $\mathrm{SO}_{2}(\mathrm{Pinteraction}=0.073)$ were observed.

In patients without CVD, there was a significant, strong 
A

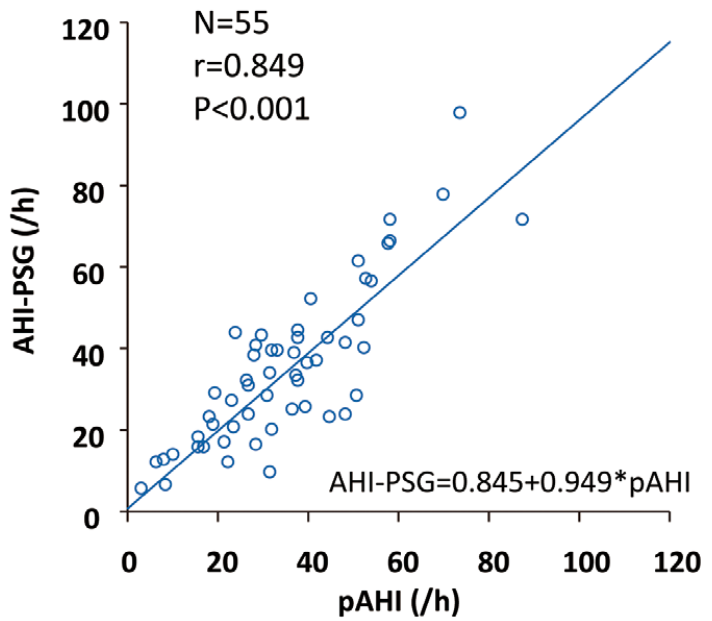

B

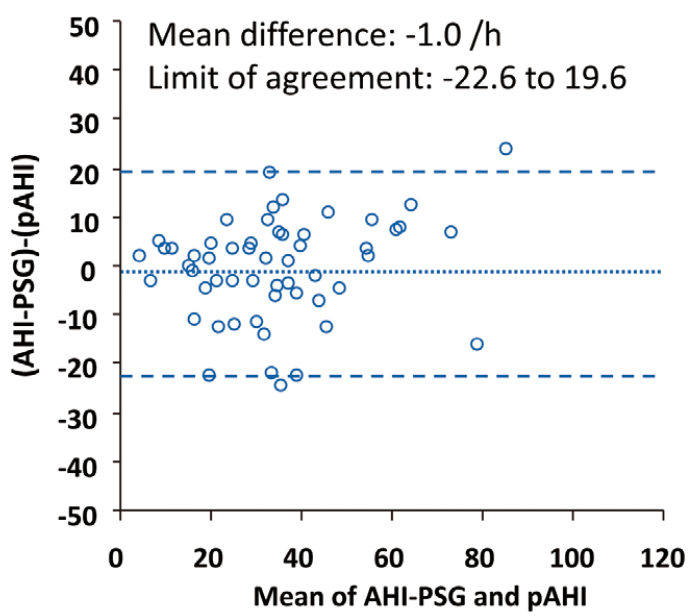

Figure 3. (A) Correlation and (B) agreement between the apnea-hypopnea index determined using a peripheral arterial tonometry-based device (pAHI) and the apnea-hypopnea index determined by polysomnography (AHI-PSG) in patients with cardiovascular disease (CVD). There was a strong correlation (A) and agreement (B) between AHI-PSG and pAHI in patients with CVD. The dotted line represents the mean difference and the large dashed lines represent the limits of agreement.

correlation ( $\mathrm{r}=0.927, \mathrm{P}<0.001$; Figure 2A) and an acceptable agreement (Figure 2B) between pAHI and AHI-PSG. The ROC analysis of patients without CVD revealed an AUC of 0.916 (standard error, $0.035 ; \mathrm{P}<0.001$ ). The best cut-off of pAHI identifying AHI-PSG $\geq 30 / \mathrm{h}$ was $27.5 / \mathrm{h}$, with a sensitivity and specificity of $87.1 \%$ and $82.4 \%$, respectively. A moderate correlation was observed between TST measured using a PAT-based device and TST measured using polysomnography $(\mathrm{r}=0.572, \mathrm{P}<0.001)$, with a mean difference of $-65.0 \mathrm{~min}$ (limits of agreement, -190.2 to $60.2 \mathrm{~min}$ ). A weak correlation between deep sleep measured using a PAT-based device and N3 sleep measured using polysomnography was observed $(\mathrm{r}=0.395, \mathrm{P}=0.002)$, with a mean difference of $-7.9 \%$ (limits of agreement, $-21.7 \%$ to $5.9 \%$ ). There was a weak correlation between REM sleep measured using a PAT-based device and R sleep measured using polysomnography $(\mathrm{r}=0.355, \mathrm{P}=0.004)$, with a mean difference of $-9.1 \%$ (limits of agreement, $-26.7 \%$ to $8.5 \%$ ). The mean $\mathrm{SO}_{2}$ measured using a PAT-based device was significantly correlated with that measured using polysomnography $(\mathrm{r}=0.803, \mathrm{P}<0.001)$, with a mean difference of $2.2 \%$ (limits of agreement, $-0.6 \%$ to $3.8 \%$ ). The minimum $\mathrm{SO}_{2}$ measured using a PAT-based device was significantly correlated with that measured using polysomnography $(\mathrm{r}=0.895, \mathrm{P}<0.001)$, with a mean difference of $-0.8 \%$ (limits of agreement, $-11.0 \%$ to $9.4 \%$ ).

In patients with CVD, there was a significant, strong correlation $(r=0.871, P<0.001$; Figure 3A), and an acceptable agreement (Figure 3B) between pAHI and AHI-PSG. The ROC analysis of patients with CVD revealed an AUC of 0.860 (standard error, $0.050 ; \mathrm{P}<0.001$ ). The best cut-off of pAHI identifying AHI-PSG $\geq 30 / \mathrm{h}$ was $32.0 / \mathrm{h}$, with a sensitivity and specificity of $76.7 \%$ and $80.0 \%$, respectively. A moderate correlation was observed between TST measured using a PAT-based device and TST measured using polysomnography $(\mathrm{r}=0.540, \mathrm{P}<0.001)$, with a mean difference of $-76.8 \mathrm{~min}$ (limits of agreement, -230.4 to
$76.8 \mathrm{~min}$ ). There was a weak correlation between deep sleep measured using a PAT-based device and N3 sleep measured using polysomnography $(\mathrm{r}=0.376, \mathrm{P}=0.007)$, with a mean difference of $-5.2 \%$ (limits of agreement, $-19.0 \%$ to $8.6 \%$ ). No correlation was found between REM sleep measured using a PAT-based device and $\mathrm{R}$ sleep measured using polysomnography $(\mathrm{r}=0.217, \mathrm{P}=0.119$; mean difference, $-5.7 \%$; limits of agreement, $-23.7 \%$ to $12.3 \%$ ). The mean $\mathrm{SO}_{2}$ measured using a PAT-based device was significantly correlated with that measured using polysomnography $(\mathrm{r}=0.916, \mathrm{P}<0.001)$, with a mean difference of $1.7 \%$ (limits of agreement, $-0.3 \%$ to $3.7 \%$ ). The minimum $\mathrm{SO}_{2}$ measured using a PAT-based device was significantly correlated with that measured using polysomnography $(\mathrm{r}=0.902, \mathrm{P}<0.001)$, with a mean difference of $-1.7 \%$ (limits of agreement, $-10.9 \%$ to $7.5 \%$ ).

\section{Discussion}

The present study provides novel insights into the clinical utility of a PAT-based device for screening and diagnosing SDB. We found a strong correlation and acceptable agreement between the pAHI and the simultaneously recorded AHI-PSG in a Japanese patient population including patients with CVD. The correlation between pAHI and AHI-PSG was similar among patients with and without CVD. The sleep parameters determined by a PATbased device differed significantly from those measured using polysomnography, but the correlation with TST measurements was acceptable and that with sleep stages was weak. We found that $\mathrm{pAHI} \geq 32.0 / \mathrm{h}$ is an indicator of severe SDB in patients with CVD. In patients without $\mathrm{CVD}$, pAHI $\geq 27.5 / \mathrm{h}$ is an indicator of severe SDB. These findings suggest that AHI determined using a PAT-based device with automatic scoring is highly reliable in Japanese patients independent of the presence of CVD. However, parameters other than AHI should be interpreted with 
caution.

Multiple studies and meta-analyses have demonstrated that the parameters determined using a PAT-based device are identical to those determined using polysomnography. ${ }^{7-10}$ However, all previous studies included populations from Western countries, and only a few studies provided comparisons between pAHI and AHI-PSG among Asian populations. ${ }^{20-22}$ In a recent study from Singapore, Gan et al reported that the recordings from a PAT-based device were comparable to those obtained from polysomnography in a patient population with suspected SDB. ${ }^{22}$ To our knowledge, no such studies with a Japanese population have been reported. Because the fit of the PAT sensor around the finger is critical, but the sensor only comes in one size and the body mass index of Japanese patients with SDB is generally less than that of SDB patients in Western countries, we believe that there is a need to conduct the same study again in Japan in order to assess whether "one-size-fits all" is true. The present study is the first to compare pAHI and AHI-PSG in a prospectively enrolled Japanese patient population from 6 centers with centralized, blinded scoring. We found a strong correlation and acceptable agreement between pAHI and AHI-PSG independent of the presence of CVD. Our results are consistent with those of previous studies. ${ }^{10,22}$

Because PAT signals are affected by changes in vascular volume detected by the finger-mounted plethysmograph, ${ }^{11}$ the altered peripheral vascular properties in patients with CVD can affect parameters derived from a PAT-based device. Kinoshita et al showed that an increased pulse wave velocity (PWV; an early sign of atherosclerosis) resulted in a weaker correlation between pAHI and AHIPSG determined on different occasions. ${ }^{12}$ The results of that study indicated that PAT-based device recordings of patients with CVD should be carefully interpreted. However, in the present study we found a strong correlation and good agreement between pAHI and AHI-PSG independent of the presence of CVD. We did not detect a significant interaction between the correlation of pAHI and AHIPSG and CVD. The strong correlation between pAHI and AHI-PSG among patients with CVD suggests that pAHI can be used to determine the severity of SDB in patients with CVD.

In addition to an alteration in the PAT signal, pAHI is derived from changes in pulse rate in response to respiratory events, ${ }^{11}$ and suppression of the pulse rate by $\beta$-blockers can affect the discrepancy between pAHI and AHI-PSG. In addition, the presence of atrial fibrillation, especially persistent/permanent atrial fibrillation, in the CVD group may have contributed to the discrepancy between pAHI and AHI-PSG. Nevertheless, it should be noted that a strong correlation and acceptable agreement were observed between pAHI and AHI-PSG in the present study, even in patients with CVD.

This study also included patients using vasodilator medications, such as ACEIs/ARBs, calcium channel blockers, and carvedilol. ${ }^{23}$ It has been reported that pAHI may not be accurate for patients using $\alpha$-blockers because these medications are known to alter the peripheral vascular tone by blocking vascular $\alpha$-adrenergic receptors, resulting in attenuated PAT signal changes of sympathetic nerve activation in response to respiratory events. ${ }^{15,24}$ Therefore, we excluded patients using $\alpha$-blockers from this study. However, we did include patients using $\alpha / \beta$-blockers (carvedilol) because this medication is frequently used to treat several types of CVD, including hypertension, and excluding these patients would not reflect the real-world clinical setting. We found that a PAT-based device could be used to screen for and diagnose SDB even in patients at risk of or already diagnosed with CVD.

Although overnight polysomnography is the standard diagnostic test for SDB,${ }^{4}$ many patients suspected of having SDB may not be able to undergo this test due to its high cost, limited access, and long waiting times. ${ }^{5}$ In addition, patients may be unwilling to undergo overnight polysomnography without objective data suspecting the presence of severe SDB. Patients with CVD and SDB are less likely to complain of excessive daytime sleepiness, which is an important SDB-related symptom indicating the need for treatment. 25-27 Therefore, providing objective data regarding SDB severity is important, particularly in patients with CVD. We found that $\mathrm{pAHI} \geq 32.0 / \mathrm{h}$ can be used as an objective guide to recommend formal polysomnography in patients with CVD.

The findings in this study indicate that the PAT-based device overestimated the TST and percentage of deep sleep and REM sleep. Sleep/wake detection is based on the assessment of movements by actigraphy, and the detection of specific sleep stages (REM and deep and light sleep) is based on the spectral components of the PAT signal. ${ }^{16-18}$ We found a moderate correlation in TST measurements between a PAT-based device and polysomnography, which is consistent with the results from a previous study in Asian patients without CVD. ${ }^{22} \mathrm{We}$ do not know why pAHI and AHI-PSG were almost the same even though TST was significantly longer with the PAT-based device than polysomnography. It is possible that apneas and hypopneas in the epoch determined as wakefulness may have contributed to this finding. Such respiratory events were omitted in the polysomnography analysis but were scored if the PATbased device determined such epochs as sleeping. However, for unknown reasons, only a weak correlation between the methods was detected for sleep staging. The PAT-based device underestimated the mean $\mathrm{SO}_{2}$ while overestimating minimum $\mathrm{SO}_{2}$, although the absolute differences have minimal clinical significance. The oxygenation parameters measured by a PAT-based device and polysomnography were more strongly correlated among patients with CVD than those without CVD, which may be due to minor differences in the detection of pulse oximetry between the 2 measurement methods.

This study is not without limitations. First, we compared the total scores of data from a PAT-based device and data from polysomnography. Data recorded from both devices for individual patients were not compared directly due to technical limitations of precise synchronization of data for each method. Therefore, our results may differ from studies that analyzed data on an epoch-by-epoch basis, ${ }^{\mathbf{1 8}, 28}$ particularly in terms of the results of sleep staging. However, we believe that the results of total score-based analyses are more generalizable in clinical settings. Second, PAT-based device recordings and polysomnography were performed simultaneously in this study. This is a strength of the study because it controls for night-to-night variation in SDB severity and sleep status, but such night-to-night variations should be considered when results from sleep studies are interpreted. In a previous study, the pAHI and AHI-PSG were determined using data from separate nights and were found to be moderately correlated, although only a few patients with ischemic heart disease and arrhythmia were 
enrolled in that study. ${ }^{12}$ Finally, the patient populations in this study, especially within the subgroups of patients with and without CVD, were relatively small.

\section{Conclusions}

In conclusion, we found that a PAT-based device provides reliable information that can be used to screen for and diagnose SDB in a Japanese patient population independent of the presence of CVD. Furthermore, we identified cut-off levels for severe SDB in patients with and without CVD. These findings may help reduce the number of patients with undiagnosed severe SDB and increase awareness of the SDB diagnosis and its treatment among physicians and patients with CVD.

\section{Acknowledgments}

None.

\section{Sources of Funding}

This study was supported by Philips. The funding source had no other roles in this study.

\section{Disclosures}

T.K. and F.K. are affiliated with a department at Juntendo University endowed by Philips, Fukuda Denshi, and ResMed. K.C. is affiliated with a department at Kyoto University endowed by Philips-Respironics, Fukuda Denshi, Fukuda Lifetec Keiji, and ResMed. The other authors have no conflicts of interest to declare.

\section{IRB Information}

This study was approved by the Juntendo University Hospital Institutional Review Board (Reference no. 14-003).

\section{Data Availability}

All deidentified participant data collected during the study will be shared. The data will be available for a period of 1 year after this article is accepted for publication. The data can be requested by emailing the corresponding author and will be shared as Excel files.

\section{References}

1. Kasai T, Floras JS, Bradley TD. Sleep apnea and cardiovascular disease: A bidirectional relationship. Circulation 2012; 126: $1495-1510$.

2. Watson NF. Health care savings: The economic value of diagnostic and therapeutic care for obstructive sleep apnea. J Clin Sleep Med 2016; 12: 1075-1077.

3. Sullivan F. Hidden health crisis costing America billions. Underdiagnosing and undertreating obstructive sleep apnea draining healthcare system. Darien, IL: American Academy of Sleep Medicine, 2016. https://aasm.org/resources/pdf/sleepapnea-economic-crisis.pdf (accessed September 8, 2020).

4. Kapur VK, Auckley DH, Chowdhuri S, Kuhlmann DC, Mehra R, Ramar K, et al. Clinical practice guideline for diagnostic testing for adult obstructive sleep apnea: An American Academy of Sleep Medicine clinical practice guideline. J Clin Sleep Med 2017; 13: $479-504$

5. Masa JF, Montserrat JM, Durán J. Diagnostic access for sleep apnea in Spain. Am J Respir Crit Care Med 2004; 170: 195; author repy $195-196$.

6. Yamada Y, Kasagi S, Tamura H, Kawana F, Tomita Y, Narui $\mathrm{K}$, et al. Clinical utility of a type 4 portable device for in-home screening of sleep disordered breathing. Ann Palliat Med 2020 Sep 10: apm-20-384. doi:10.21037/apm-20-384.

7. Bar A, Pillar G, Dvir I, Sheffy J, Schnall RP, Lavie P. Evaluation of a portable device based on peripheral arterial tone for unattended home sleep studies. Chest 2003; 123: 695-703.

8. Yuceege M, Firat H, Demir A, Ardic S. Reliability of the WatchPAT 200 in detecting sleep apnea in highway bus drivers. J Clin Sleep Med 2013; 9: 339-344.

9. Onder NS, Akpinar ME, Yigit O, Gor AP. Watch peripheral arterial tonometry in the diagnosis of obstructive sleep apnea: Influence of aging. Laryngoscope 2012; 122: 1409-1414

10. Yalamanchali S, Farajian V, Hamilton C, Pott TR, Samuelson CG, Friedman M. Diagnosis of obstructive sleep apnea by peripheral arterial tonometry: Meta-analysis. JAMA Otolaryngol Head Neck Surg 2013; 139: 1343-1350.

11. Schnall RP, Shlitner A, Sheffy J, Kedar R, Lavie P. Periodic, profound peripheral vasoconstriction: A new marker of obstructive sleep apnea. Sleep 1999; 22: 939-946.

12. Kinoshita T, Yahaba M, Terada J, Matsumura T, Sakurai Y, Nagashima $K$, et al. Impact of arterial stiffness on WatchPAT variables in patients with obstructive sleep apnea. $J$ Clin Sleep Med 2018; 14: 319-325.

13. Berry RB, Brooks R, Gamaldo CE, Harding SM, Lloyd RM, Marcus CL, et al. The AASM Manual for the Scoring of Sleep and Associated Events: Rules, Terminology and Technical Specifications, Version 2.2. Darien, IL: American Academy of Sleep Medicine, 2015

14. American Academy of Sleep Medicine. International Classification of Sleep Disorders. 3rd ed. Darien, IL: American Academy of Sleep Medicine, 2014.

15. Zou D, Grote L, Eder DN, Peker Y, Hedner J. Obstructive apneic events induce alpha-receptor mediated digital vasoconstriction. Sleep 2004; 27: 485-489.

16. Hedner J, Pillar G, Pittman SD, Zou D, Grote L, White DP. A novel adaptive wrist actigraphy algorithm for sleep-wake assessment in sleep apnea patients. Sleep 2004; 27: 1560-1566.

17. Herscovici S, Pe'er A, Papyan S, Lavie P. Detecting REM sleep from the finger: An automatic REM sleep algorithm based on peripheral arterial tone (PAT) and actigraphy. Physiol Meas 2007; 28: 129-140.

18. Bresler M, Sheffy K, Pillar G, Preiszler M, Herscovici S. Differentiating between light and deep sleep stages using an ambulatory device based on peripheral arterial tonometry. Physiol Meas 2008; 29: $571-584$.

19. Akobeng AK. Understanding diagnostic tests 3: Receiver operating characteristic curves. Acta Paediatr 2007; 96: 644-647.

20. Choi JH, Kim EJ, Kim YS, Choi J, Kim TH, Kwon SY, et al. Validation study of portable device for the diagnosis of obstructive sleep apnea according to the new AASM scoring criteria: Watch-PAT 100. Acta Otolaryngol 2010; 130: 838-843.

21. Li W, Wang R, Huang D, Liu X, Jin W, Yang S. Assessment of a portable monitoring device WatchPAT 200 in the diagnosis of obstructive sleep apnea. Eur Arch Otorhinolaryngol 2013; 270: 3099-3105.

22. Gan YJ, Lim L, Chong YK. Validation study of WatchPat 200 for diagnosis of OSA in an Asian cohort. Eur Arch Otorhinolaryngol 2017; 274: 1741-1745.

23. Pedersen ME, Cockcroft JR. The vasodilatory beta-blockers. Curr Hypertens Rep 2007; 9: 269-277.

24. Grote L, Zou D, Kraiczi H, Hedner J. Finger plethysmography: A method for monitoring finger blood flow during sleep disordered breathing. Respir Physiol Neurobiol 2003; 136: 141-152.

25. Arzt M, Young T, Finn L, Skatrud JB, Ryan CM, Newton GE, et al. Sleepiness and sleep in patients with both systolic heart failure and obstructive sleep apnea. Arch Intern Med 2006; 166: $1716-1722$

26. Albuquerque FN, Calvin AD, Sert Kuniyoshi FH, Konecny T, Lopez-Jimenez F, Pressman GS, et al. Sleep-disordered breathing and excessive daytime sleepiness in patients with atrial fibrillation. Chest 2012; 141: 967-973.

27. Baniak LM, Chasens ER, Luyster FS, Strollo PJ Jr, Thunstrom E, Peker Y. Obstructive sleep apnea and self-reported functional impairment in revascularized patients with coronary artery disease in the RICCADSA trial. Sleep Breath 2018; 22: 1169-1177.

28. Hedner J, White DP, Malhotra A, Herscovici S, Pittman SD, Zou D, et al. Sleep staging based on autonomic signals: A multi-center validation study. J Clin Sleep Med 2011; 7: 301-306. 